\title{
Simulation of swift boron clusters traversing amorphous carbon foils
}

\author{
Santiago Heredia-Avalos, ${ }^{1}$ Isabel Abril, ${ }^{2}$ Cristian D. Denton, ${ }^{2}$ and Rafael Garcia-Molina ${ }^{1}$ \\ ${ }^{1}$ Departamento de Física - CIOyN, Universidad de Murcia, Apartado 4021, E-30080 Murcia, Spain \\ ${ }^{2}$ Departament de Física Aplicada, Universitat d'Alacant, Apartat 99, E-03080 Alacant, Spain
}

(Received 1 June 2006; published 9 January 2007)

\begin{abstract}
We use a simulation code to study the interaction of swift boron clusters $\left(\mathrm{B}_{n}{ }^{+}, n=2-6,14\right)$ with amorphous carbon foils. We analyze different aspects of this interaction, such as the evolution of the cluster structure inside the target, the energy and angle distributions at the detector or the stopping power ratio. Our simulation code follows in detail the motion of the cluster fragments through the target and in the vacuum until reaching a detector, taking into account the following interactions: (i) wake force, (ii) Coulomb repulsion among cluster fragments, (iii) stopping force, and (iv) elastic scattering with the target nuclei. Electron capture and loss by each fragment is also included in the code, affecting the above-mentioned interactions. The clusters size grows inside the foil due mainly to the Coulomb explosion but this increase is less pronounced in the plane transversal to the beam direction because of the alignment effect of the wake forces. We obtain an enhancement of the stopping power ratio that increases with the projectile energy and with the number of molecular constituents. Our results agree very well with the available experimental data for the thicker foils $\left(\gtrsim 10 \mu \mathrm{g} / \mathrm{cm}^{2}\right)$ and are compatible (within the experimental error bars) for the thinner foils.
\end{abstract}

DOI: 10.1103/PhysRevA.75.012901

PACS number(s): 34.50.Bw, 36.40.- c, 61.48.+c

\section{INTRODUCTION}

Beams of swift charged particles have been widely used both to analyze materials or to modify their properties [1,2]. Ion beams are commonly applied in different areas such as microelectronics, target characterization, radiation therapy or protection against radiation [3-6]. In particular the use of molecular and cluster ion beams has been proposed in the last years because of their ability to deposit energy in a small region of the target and hence the interest for inertial confinement fusion studies [7-9].

When a swift cluster penetrates a solid, the energy it loses interacting with the solid can be rather different than the energy lost by the cluster fragments considered independently. This is a consequence of the so-called "vicinage effect" that is due to the electronic perturbation in the solid caused by the motion of each cluster fragment, which in turn affects the motion of the other components of the cluster $[10]$.

The stopping power ratio, defined as the stopping power of a material for the whole cluster divided by the sum of the stopping powers for all the cluster components considered independently, is used to quantify the vicinage effects in the energy loss. Experimental stopping power ratios up to $\sim 1.5$ have been reported in the literature [11-18]. The state of the art concerning this subject can be consulted in the recent review by Arista [10]. Aside from the energy loss, other magnitudes, such as the charge state of the fragments, are known to undergo vicinage effects $[19,20]$.

In this paper we focus on the study of the interaction of swift boron clusters $\left(\mathrm{B}_{n}^{+}, n=2-6,14\right)$ with amorphous carbon foils, using a simulation code that improves previous analytical studies that only provided average magnitudes $[21,22]$. The different shapes and sizes of the clusters allow us to analyze the vicinage effects and the evolution of the cluster structure in rather different situations.

The paper is organized as follows. In Sec. II the main ingredients of our simulation code are presented. Section III is devoted to show the main results and the discussion about them. The conclusions of our study appear in Sec. IV.

Atomic units are employed throughout this work, except where otherwise stated.

\section{SIMULATION CODE}

When a cluster penetrates a solid it loses its binding electrons in the first atomic layers. From that moment, the cluster can be considered as a group of atoms with a certain initial spatial distribution moving through the target in a correlated way.

We need to specify the initial geometrical structure of the cluster in order to describe the evolution with time of the fragments resulting from the cluster dissociation. In Fig. 1 we show the initial geometrical structures of the different

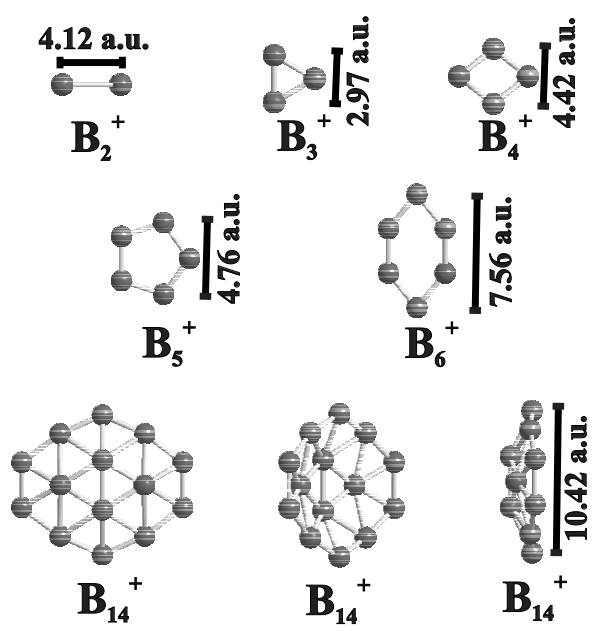

FIG. 1. Structure of the boron clusters before entering the target [23]. Different orientations are shown for the $\mathrm{B}_{14}{ }^{+}$cluster in order to clarify its shape. 
boron clusters analyzed in this paper, which were obtained from ab initio calculations [23]. All the clusters have planar structures, except the $\mathrm{B}_{14}{ }^{+}$molecular ion, which has a bowltype shape; so to clearly show its structure it is depicted with different orientations in the figure.

The boron atoms resulting from the cluster dissociation (from now on the fragments) capture or lose electrons from the target, acquiring different charge states during their motion. They also suffer forces inside the target mainly caused by their interaction among themselves and with the target electrons and nuclei. Most of these interactions, except the scattering with the target nuclei, depend on the charge state of the fragments.

Our simulation code has been described elsewhere [24-26] and it uses a finite difference algorithm [27] to calculate numerically the trajectories of all the fragments through the foil and in the vacuum until reaching a detector. It includes in a realistic way all the interactions just mentioned (including the charge exchange of the fragments). In addition, our simulation takes into account that usually the clusters impinge on the target randomly oriented with respect to the beam direction, except when otherwise stated.

By keeping track of the coordinates and velocities of the fragments we are able to obtain several magnitudes inside the foil, just at the exit of the foil or asymptotically when reaching a detector, such as energy distributions, exit angle distributions, charge state distributions or even interatomic distance distributions.

\section{A. Charge exchange}

As previously stated each fragment resulting from the dissociation of the cluster captures or loses electrons in the target, changing its initial charge state. Once the charge equilibrium is attained the fragment continues changing its charge state, but the probability to find the fragment in each charge state, i.e., the charge fraction at a given projectile velocity, remains unaltered. In our simulation we decide the distances between electron capture or loss events as well as their probability for the capture and loss cross sections [28]; the required equilibrium charge fractions are given by the CasP code $[29,30]$. These charge fractions are shown in Fig. 2 for boron projectiles in amorphous carbon foils, as a function of the incident energy. We assume that the charge state of each atomic ion resulting from the molecular dissociation is identical to that corresponding to the same atomic (but isolated) ion. Using an analytical treatment [20,31], we have estimated that vicinage effects in the charge state have no practical consequences in the energy loss of the fragments.

Taking into account the charge fractions given by the CasP code $[29,30]$ and using our simulation code, we can obtain the evolution of the mean charge for the boron ions as a function of the depth inside the target; we show in Fig. 3 the mean charge state for two different projectile incident energies. As can be clearly seen the mean charge evolves from the initial charge $(+1)$ until the charge equilibrium is completely reached at target depths larger than $1 \mu \mathrm{g} / \mathrm{cm}^{2}$. The depth at which the charge equilibrium is reached depends on the initial projectile energy.

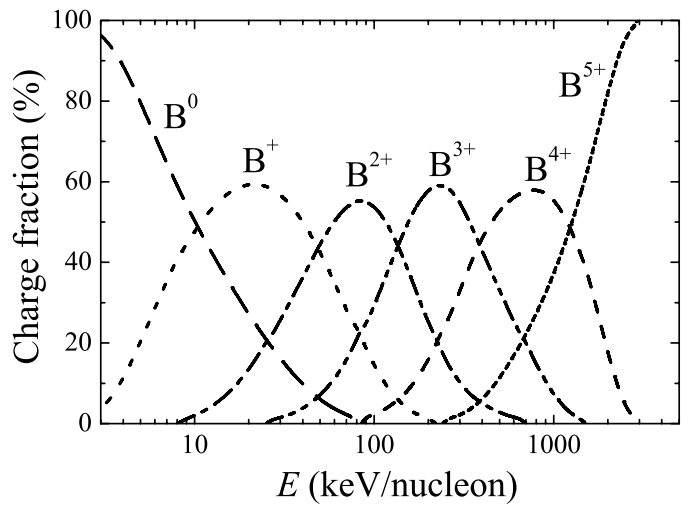

FIG. 2. Charge fractions of boron projectiles inside an amorphous carbon foil once the charge equilibrium has been reached, as a function of the projectile energy. They were obtained using the CasP code [29,30].

\section{B. Interactions with the target electrons}

The interaction of the cluster fragments with the target electrons is the main responsibility for their energy losses, at least in the energy range we are considering $\left(E_{0}>75 \mathrm{keV} /\right.$ nucleon). The energy loss of each fragment depends on its charge state, velocity, and the response of the target. The electronic energy loss is included in our study using the dielectric formalism $[32,33]$ with a realistic description of the target [34-36]. The stopping power of the target for a given fragment $i$ moving with velocity $v_{i}$ through the target, or the mean energy loss $\left\langle\Delta E_{i}\right\rangle$ per unit path length $\Delta x$, is given by

$$
\begin{aligned}
S_{p, i}(v) & =-\frac{\left\langle\Delta E_{i}\right\rangle}{\Delta x} \\
& =\frac{2}{\pi v_{i}^{2}} \int_{0}^{\infty} \frac{d k}{k}\left[Z_{i}-G_{i}(k)\right]^{2} \int_{0}^{k v_{i}} d \omega \omega \operatorname{Im}\left[\frac{-1}{\epsilon(k, \omega)}\right] .
\end{aligned}
$$

$k$ and $\omega$ are the momentum and energy transferred to the target electrons, respectively. The stopping power depends on the response of the target material through the energy loss

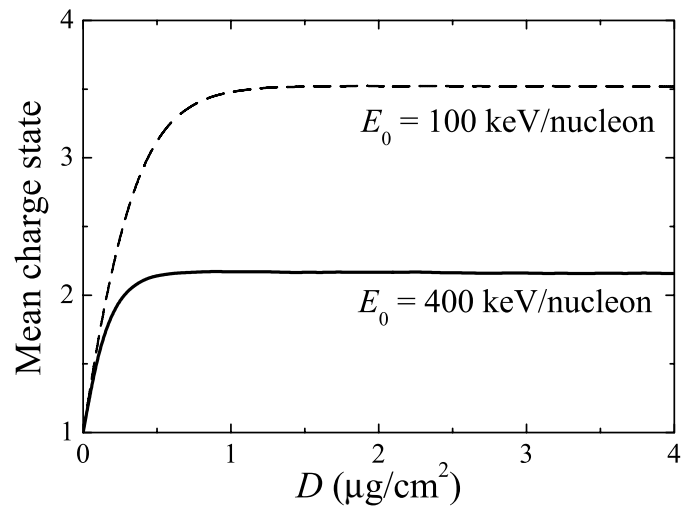

FIG. 3. Mean charge state that the initial $\mathrm{B}^{+}$ions acquire inside an amorphous carbon foil, as a function of the target depth. The projectile incident energy is indicated for each curve. 
function (ELF), $\operatorname{Im}[-1 / \epsilon(k, \omega)]$, and on the projectile charge density by means of $Z_{i}-G_{i}(k), Z_{i}$ and $G_{i}(k)$ being the atomic number and the Fourier transform of the electronic density of the $i$ fragment, respectively. $G_{i}(k)$ was included through the statistical Brandt-Kitagawa model [37].

The ELF of a material contains information about the electromagnetic excitations that a target can sustain and can be properly modeled by separating the contribution coming from the inner electrons of the target ( $K$ shell in the case of amorphous carbon) and the outer electrons [34-36]. The contribution from the outer electrons is taken into account by fitting the available experimental optical $(k=0)$ data [38] by means of a sum of Mermin-type energy loss functions,

$$
\operatorname{Im}\left[\frac{-1}{\epsilon(k=0, \omega)}\right]_{\text {outer }}=\sum_{i} A_{i} \operatorname{Im}\left[\frac{-1}{\epsilon_{M}\left(\omega_{i}, \gamma_{i} ; k=0, \omega\right)}\right],
$$

where $\epsilon_{M}$ is a Mermin-type dielectric function [39], with $\omega_{i}$, $\gamma_{i}$, and $A_{i}$ being fitting parameters that correspond to the position, width, and relative weight of each Mermin-type ELF of the energy loss spectrum.

On the other hand, the excitation of the inner-shell electrons of the target atoms are described by means of generalized oscillator strengths (GOS) in the hydrogenic approach, which are related with the ELF according to $[40,41]$

$$
\operatorname{Im}\left[\frac{-1}{\epsilon(k, \omega)}\right]_{\mathrm{inner}}=\frac{2 \pi^{2} \mathcal{N}}{\omega} \sum_{n \ell} \frac{d f_{n \ell}(k, \omega)}{d \omega},
$$

where $\mathcal{N}$ is the atomic density of the target and $d f_{n \ell}(k, \omega) / d \omega$ is the GOS of the $(n, \ell)$ subshell. The summation is extended over all inner subshells of the target atoms. Of course, the ionization of a given subshell can only take place if the energy transfer $\omega$ is larger than a threshold energy.

The resulting ELF must verify the $f$-sum rule [41], that is, the effective number of excited electrons per atom when $\omega$ $\rightarrow \infty$ tends to the total number of electrons per atom $[34,42]$. Moreover, we also calculate the mean excitation energy $I$ of each target [43] as an additional checking of our fitted ELF, obtaining a good agreement $[44,45]$ with the experimental data [46].

The statistical fluctuations around the mean energy loss are also taken into account through the energy loss straggling, defined in the dielectric formalism as

$$
\begin{aligned}
\Omega_{i}^{2}(v) & =\frac{\left\langle\Delta E_{i}^{2}\right\rangle-\left\langle\Delta E_{i}\right\rangle^{2}}{\Delta x} \\
& =\frac{2}{\pi v_{i}^{2}} \int_{0}^{\infty} \frac{d k}{k}\left[Z_{i}-G_{i}(k)\right]^{2} \int_{0}^{k v_{i}} d \omega \omega^{2} \operatorname{Im}\left[\frac{-1}{\epsilon(k, \omega)}\right] .
\end{aligned}
$$

In this way our code considers that the stopping power can fluctuate around the mean stopping power, given by $S_{p, i}$, with a Gaussian distribution whose width depends on the energy loss straggling $\Omega_{i}^{2}$.

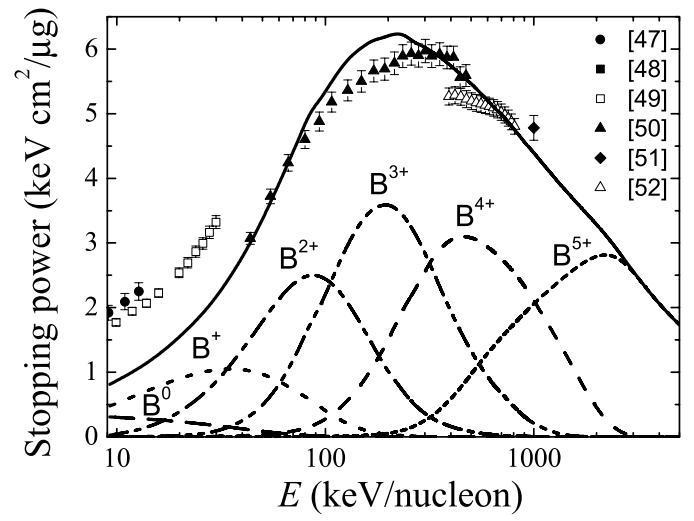

FIG. 4. Stopping power of amorphous carbon for a boron beam (incident as $\mathrm{B}^{+}$), as a function of the incident energy (solid line). The discontinuous lines represent the contribution to the stopping power for the different charge states the boron projectile can attain inside the target. Symbols represent the available experimental results $[47-52]$.

In order to check the suitability of this model to describe the mean stopping power of amorphous carbon for $\mathrm{B}^{+}$ions, we show in Fig. 4 the corresponding stopping power $S_{p, i}$ as a function of the incident energy of the projectile. The comparison of our calculations with the available experimental data [47-52] is reasonably good in the energy range considered in this work $\left(E_{0}=75-400 \mathrm{keV} /\right.$ nucleon $)$, i.e., around the stopping power maximum. The discontinuous curves represent the contributions of the different charge fractions to the stopping power. At low energies nonlinear effects arise and so the dielectric formalism is no longer suitable. It is worth to mention that the main contribution to the energy loss of swift projectiles comes from the outer target electrons.

The good comparison between the experimental data at low energies and the results obtained from the dielectric formalism (a linear response theory), could be understood by the fact that in such energy range the dominant charge states correspond to dressed ions, giving rise to a weak perturbation in the solid. All the same, nonlinear contributions (Barkas and Bloch terms) should be taken into account in a more detailed treatment of the energy loss of ions [33], but this goes beyond the purpose of the present work, whose aim is the analysis of the vicinage effects in the energy loss of clusters.

\section{Interactions with the target nuclei}

The interactions of the projectile with the target nuclei contribute slightly to the energy loss, however it is the main responsibility of the angular spread for isolated swift atomic ions. The multiple elastic scattering with the target nuclei is incorporated in our code via a Monte Carlo algorithm $[28,53]$ that, according to the target atomic density and the interaction potential between the fragments and the target atoms, changes the direction of motion of each fragment after a certain distance. We have used the universal ZBL potential [54] to describe the interaction between boron and carbon cores. The possible correlation between the scattering 
of different fragments of the cluster has not been included in our code because its effect has been found to be negligible in amorphous targets [55].

\section{Interactions among the cluster fragments}

The fragments resulting from the cluster dissociation interact between them through a screened Coulomb potential, which can be decomposed in (i) pure Coulomb repulsion forces that tend to increase the interatomic distances between the charged fragments both inside and outside the target, i.e., Coulomb explosion, and (ii) forces due to the electromagnetic excitations induced in the target electrons, the so-called wake forces, which are asymmetric and only appear inside the solid. As a matter of fact, the wake forces are not really a force exerted by a particle of the cluster on another one. The wake forces are due to the electronic density induced in the medium by a swift charge, which affect other charged particles that are in its neighborhood. In this sense these forces do not need to obey Newton's third principle. The wake force exerted by a fragment $i$ on fragment $j$ is located in a plane defined by both particles and the velocity of $i$; the dielectric formalism predicts that the force component parallel to the direction of motion of the $i$ fragment takes the form [56]

$$
\begin{aligned}
F_{i \rightarrow j, z}= & \frac{2}{\pi v_{i}^{2}} \int_{0}^{\infty} \frac{d k}{k}\left[Z_{i}-G_{i}(k)\right]\left[Z_{j}-G_{j}(k)\right] \\
& \times \int_{0}^{k v_{i}} d \omega \omega J_{0}\left(\rho_{i j} \sqrt{k^{2}-\omega^{2} / v_{i}^{2}}\right) \\
& \times\left\{\sin \left(\frac{\omega z_{i j}}{v_{i}}\right) \operatorname{Re}\left[\frac{1}{\epsilon(k, \omega)}-1\right]\right. \\
& \left.+\cos \left(\frac{\omega z_{i j}}{v_{i}}\right) \operatorname{Im}\left[\frac{1}{\epsilon(k, \omega)}-1\right]\right\},
\end{aligned}
$$

and the force component perpendicular to the direction of motion of the $i$ fragment is defined as

$$
\begin{aligned}
F_{i \rightarrow j, \rho}= & \frac{2}{\pi v_{i}} \int_{0}^{\infty} \frac{d k}{k}\left[Z_{i}-G_{i}(k)\right]\left[Z_{j}-G_{j}(k)\right] \\
& \times \int_{0}^{k v_{i}} d \omega J_{1}\left(\rho_{i j} \sqrt{k^{2}-\omega^{2} / v_{i}^{2}}\right) \sqrt{k^{2}-\omega^{2} / v_{i}^{2}} \\
& \times\left\{\cos \left(\frac{\omega z_{i j}}{v_{i}}\right) \operatorname{Re}\left[\frac{1}{\epsilon(k, \omega)}-1\right]\right. \\
& \left.-\sin \left(\frac{\omega z_{i j}}{v_{i}}\right) \operatorname{Im}\left[\frac{1}{\epsilon(k, \omega)}-1\right]\right\},
\end{aligned}
$$

where $J_{0}$ and $J_{1}$ are the Bessel functions of zero and first order, respectively, and $z_{i j}$ and $\rho_{i j}$ are the relative distance between both particles in the directions parallel $z$ and perpendicular $\rho$, respectively, to the movement of the $i$ fragment.

We have depicted in Fig. 5 the components of the wake force generated by a $400 \mathrm{keV} /$ nucleon $\mathrm{B}^{3+}$ ion and acting over a $\mathrm{B}^{4+}$ ion in the directions defined by the movement of

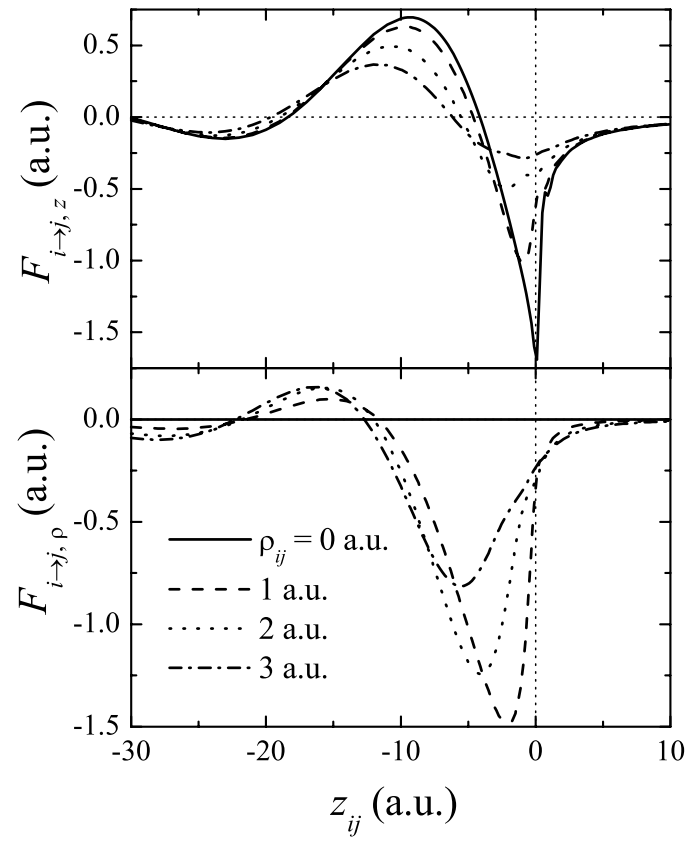

FIG. 5. Wake force generated by a $400 \mathrm{keV} /$ nucleon $\mathrm{B}^{3+}$ ion and acting on $\mathrm{a}^{4+}$ ion, both moving through an amorphous carbon target. We have represented the components of the force in the directions parallel and perpendicular to the movement direction of the $\mathrm{B}^{3+}$ ion, as a function of the relative distance between both particles.

the $\mathrm{B}^{3+}$ ion, as a function of the relative distance between both fragments. We have chosen $\mathrm{B}^{3+}$ and $\mathrm{B}^{4+}$ ions because they are the most probable charge states for this incident energy. Normally the direction of the movement of any two fragments is approximately the same. Therefore $F_{z}$ affects the energy loss of the $j$ fragment giving rise to the vicinage effects already mentioned. On the other side, $F_{\rho}$ tends to reduce the separation $\rho_{i j}$ in the direction perpendicular to the motion of the leading particle, aligning the $j$ fragment towards the direction of motion of the $i$ fragment. Both components of the wake force are much more important if the $j$ particle is behind the $i$ particle and become negligible if both particles are far away. These wake forces do not change when using a Hartree-Fock description of the projectile electronic density, instead of the Brandt-Kitagawa model.

\section{RESULTS AND DISCUSSION}

Our simulation code allows us to investigate the distortion of a swift boron cluster inside an amorphous carbon target, which is important to determine the resulting energy loss of the fragments. This distortion is illustrated in Fig. 6, where we observe how a $400 \mathrm{keV} /$ nucleon $\mathrm{B}_{14}{ }^{+}$cluster evolves with the time it spends inside the foil. The initial orientation of the $\mathrm{B}_{14}{ }^{+}$cluster, defined by the plane of its perimeter, is (a) perpendicular to the target surface and (b) parallel to it; the numbers between parentheses denote the cluster size in the direction perpendicular to the velocity. Obviously the Coulomb repulsion among the fragments tends to separate them, so they become uncorrelated at large times. However, 


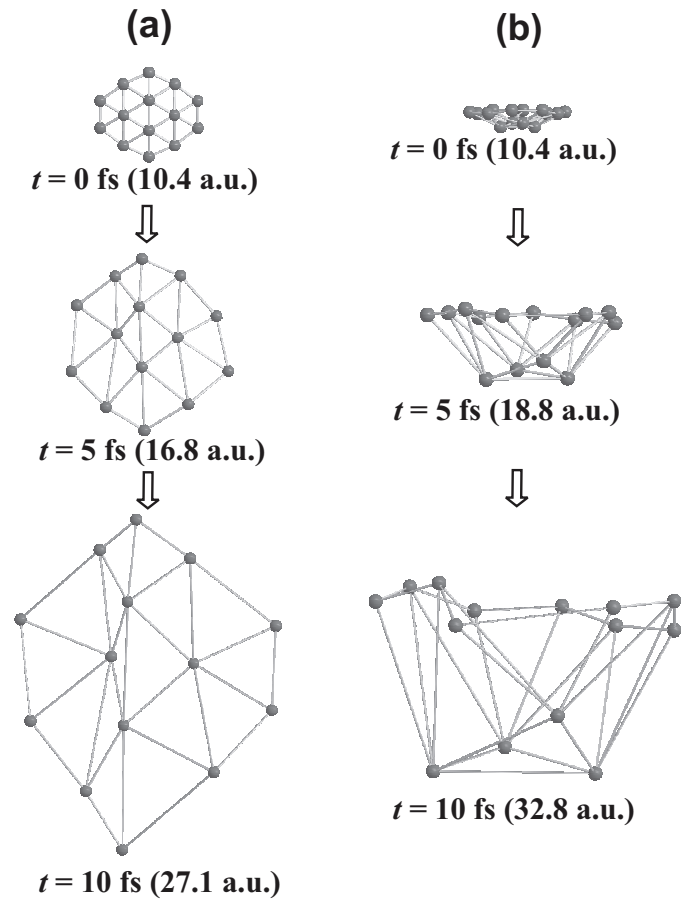

FIG. 6. Time evolution of a $400 \mathrm{keV} /$ nucleon $\mathrm{B}_{14}{ }^{+}$ion when the cluster incides (a) perpendicular or (b) parallel to the surface of an amorphous carbon target. The arrows represent the direction of motion and the numbers in parentheses are the size of the cluster in the transversal direction to the motion, at the indicated time.

it is interesting to note that the growth of the cluster size is larger in the direction of motion than in the transversal direction. The explanation of this effect is due to the wake forces that tend to align the fragments toward the direction of motion, in opposition to the effect of the Coulomb explosion. This is more pronounced when the initial orientation of the cluster is perpendicular to the surface, because in this case it is more probable to find one fragment behind another. This effect can be observed more easily in Fig. 7, where we show the evolution of the mean interatomic distance $\langle d\rangle$ for the $\mathrm{B}_{14}{ }^{+}$cluster as a function of the target thickness $D ;\langle d\rangle$ is defined as the average of the interatomic distances between all the fragments that constitute the cluster. In the picture we have also represented the components of $\langle d\rangle$ in the direction of motion of the cluster, $\langle z\rangle$, and in the transversal direction, $\langle\rho\rangle$. Clearly $\langle\rho\rangle$ grows much slower than $\langle z\rangle$ due to the inhibition of the Coulomb explosion in the transversal direction because of the wake forces. We have checked that the other $\mathrm{B}_{n}{ }^{+}$clusters also experience the same effect.

We show in Fig. 8 the energy distributions of the cluster fragments $\left(\mathrm{B}_{n}{ }^{+}, n=2-6,14\right)$ after traversing a $2 \mu \mathrm{g} / \mathrm{cm}^{2}$ thick carbon foil and reaching a detector with $0.5^{\circ}$ angle resolution. The incident energy of the clusters are (a) $E_{0}$ $=400 \mathrm{keV} /$ nucleon and (b) $E_{0}=100 \mathrm{keV} /$ nucleon. We have omitted the curves corresponding to $\mathrm{B}_{4}{ }^{+}$and $\mathrm{B}_{5}{ }^{+}$for clarity reasons; such calculations do not report any particular feature compared with those shown in the figure. No significative changes in these energy distributions were found when the initial molecular vibrations of the cluster were included in the simulation, as long as the mean interatomic distances do not change.

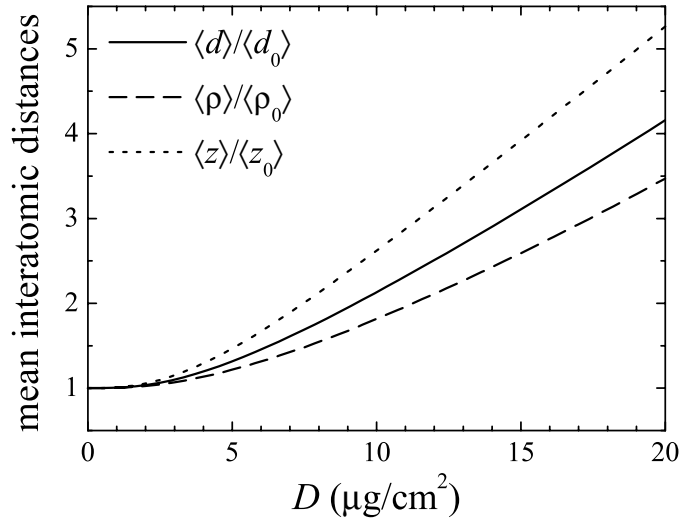

FIG. 7. Normalized mean interatomic distance $\left(\langle d\rangle /\left\langle d_{0}\right\rangle\right)$ of a $400 \mathrm{keV} /$ nucleon $\mathrm{B}_{14}{ }^{+}$molecular ion as a function of the depth in an amorphous carbon target. We represent also the components of $\langle d\rangle /\left\langle d_{0}\right\rangle$ in the direction of motion $\left(\langle z\rangle /\left\langle z_{0}\right\rangle\right)$ and in the transversal direction $\left(\langle\rho\rangle /\left\langle\rho_{0}\right\rangle\right)$. The initial mean interatomic distance and its components are $\left\langle d_{0}\right\rangle,\left\langle\rho_{0}\right\rangle$, and $\left\langle z_{0}\right\rangle$, respectively.

Through the simulations it is possible to find out the relative contributions, due to the different interactions previously described, to the energy distributions. In general terms, the Coulomb explosion is the main responsible for the width of the energy distributions. This explosion, which in the cases shown in figure 8 occur mostly in the vacuum, tends to accelerate the leading fragments and decelerate the trailing ones. The energy distributions are broader as the number of fragments $n$ increases because the available initial Coulomb potential energy is larger. The net effect of the wake force is to add an extra energy loss to the trailing fragments (those with lower energies in the distribution), widening the distri-

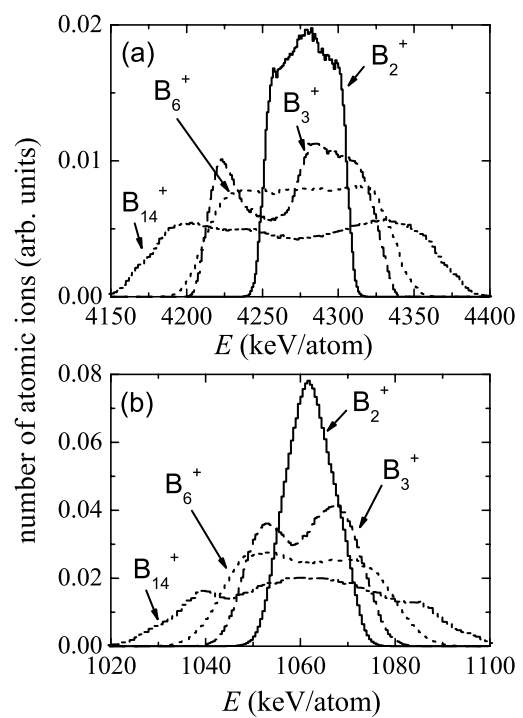

FIG. 8. Energy distributions of the ions dissociated from a $\mathrm{B}_{n}{ }^{+}$ molecular ion $\left(n=2,3,6\right.$, and 14) after traversing a $2 \mu \mathrm{g} / \mathrm{cm}^{2}$ thick carbon foil and reaching a $0.5^{\circ}$ angle detector, for an incident energy of (a) $E_{0}=400 \mathrm{keV} /$ nucleon and (b) $E_{0}=100 \mathrm{keV} /$ nucleon. All the distributions are normalized to unity area. The parameters characterizing the system were taken according to the experiments of Ref. [17]. 


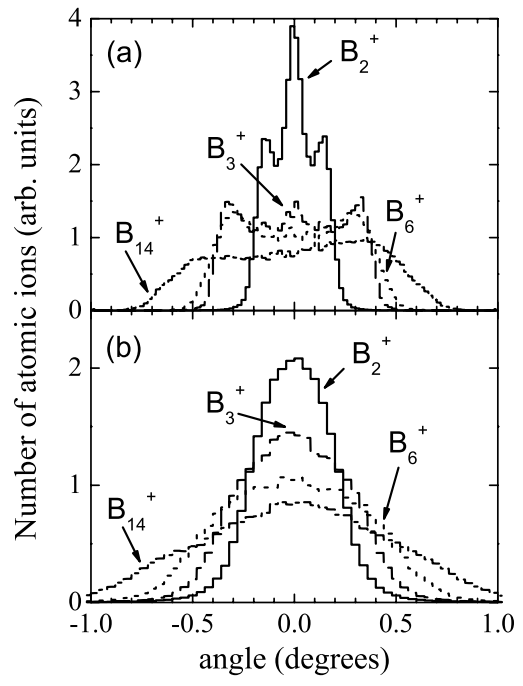

FIG. 9. Exit angle distributions of the ions dissociated from a $\mathrm{B}_{n}{ }^{+}$molecular ion $\left(n=2,3,6\right.$, and 14) after traversing a $2 \mu \mathrm{g} / \mathrm{cm}^{2}$ thick amorphous carbon foil, for an incident energy of (a) $E_{0}$ $=400 \mathrm{keV} /$ nucleon and (b) $E_{0}=100 \mathrm{keV} /$ nucleon. All the distributions are normalized to unity area. The parameters characterizing the system were taken according to the experiments of Ref. [17].

butions in that region. The elastic collisions with the target nuclei are practically irrelevant for the energy distributions.

The sharp shape of the $\mathrm{B}_{2}{ }^{+}$fragments' energy distribution, in relation to the rest of the clusters, is due to the large value of the initial interatomic distance of the cluster (see Fig. 1), which gives rise to a weak Coulomb explosion; so the difference between the energy of the leading and the trailing fragments is quite small. The case of $\mathrm{B}_{3}{ }^{+}$is special because the fragments are initially very close and so the effect of the Coulomb explosion and the wake forces are stronger, giving rise to a clear two peaked distribution. On the contrary, the $\mathrm{B}_{14}{ }^{+}$energy distributions are the broadest ones due to the high packing level of the initial cluster structure, which is responsible for a strong Coulomb explosion.

The calculated angular distributions that the cluster fragments have at the detector are depicted in Fig. 9. They correspond to distributions after traversing a $2 \mu \mathrm{g} / \mathrm{cm}^{2}$ thick carbon foil for an incident energy of (a) $E_{0}$ $=400 \mathrm{keV} /$ nucleon and (b) $E_{0}=100 \mathrm{keV} /$ nucleon. As in Fig. 8 we have omitted the curves corresponding to $\mathrm{B}_{4}{ }^{+}$and $\mathrm{B}_{5}{ }^{+}$ for clarity reasons. Anyway these curves present a behavior similar to that of the $\mathrm{B}_{6}{ }^{+}$cluster. The Coulomb explosion is responsible again for most of the width of the angular distributions and so they are broader as the number $n$ of cluster fragments increases. The wake forces give rise to the peak appearing at $0^{\circ}$ due to the alignment effect on the trailing fragments. At $E_{0}=100 \mathrm{keV} /$ nucleon the increasing effect of the elastic collisions tends to wash out this alignment giving rise to nearly Gaussian distributions. This contribution of the elastic collisions to the angular spread makes the distributions broader at lower energies.

Finally we investigate the vicinage effects in the energy loss of boron clusters in amorphous carbon. In order to quantify this effect we use the stopping power ratio $R$, which in the case of $\mathrm{B}_{n}{ }^{+}$clusters takes the form

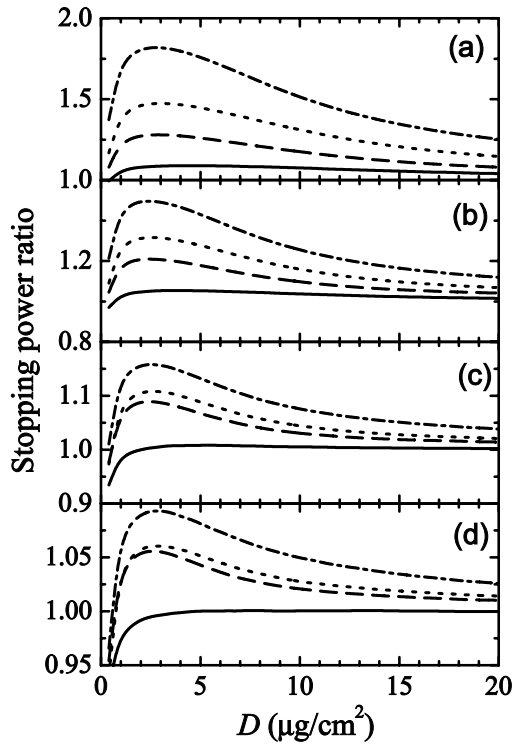

FIG. 10. Stopping power ratio for $\mathrm{B}_{n}{ }^{+}$molecular ions $(n=2,3$, 6 , and 14) as a function of the thickness of an amorphous carbon foil, for several incident energies: (a) $E_{0}=400 \mathrm{keV} /$ nucleon, (b) $E_{0}=225 \mathrm{keV} /$ nucleon, (c) $E_{0}=100 \mathrm{keV} /$ nucleon, and (d) $E_{0}$ $=74 \mathrm{keV} /$ nucleon. The different type of lines indicates the number $n$ of atomic ions that constitutes the molecular ion: (-) $n=2,(--)$ $n=3,(\cdots) n=6$, and (-.-) $n=14$.

$$
R=\frac{\Delta E\left(\mathrm{~B}_{n}^{+}\right)}{n \Delta E\left(\mathrm{~B}^{+}\right)}
$$

where $\Delta E\left(\mathrm{~B}_{n}^{+}\right)$is the energy loss of the whole boron cluster and $\Delta E\left(\mathrm{~B}^{+}\right)$is that corresponding to an isolated $\mathrm{B}^{+}$ion, both moving with identical velocity through the same target thickness. $R \neq 1$ reflects the existence of vicinage effects in the energy loss. We have calculated $R$ as a function of the target

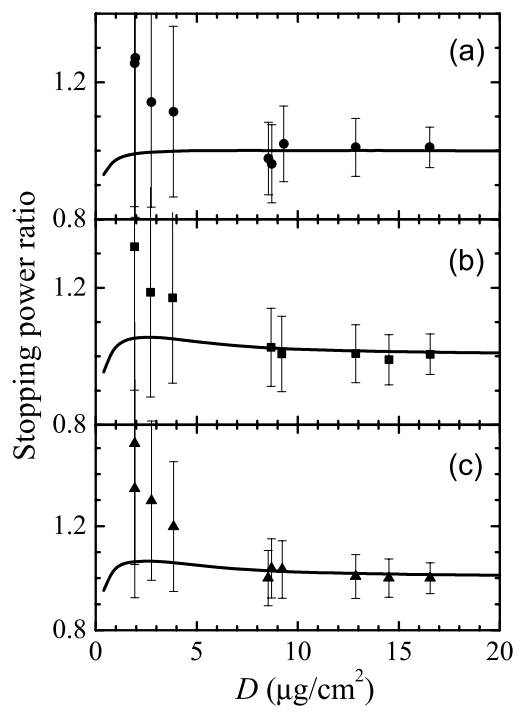

FIG. 11. Stopping power ratio of amorphous carbon for a $74 \mathrm{keV} /$ nucleon $\mathrm{B}_{n}{ }^{+}$molecular ion, as a function of the target thickness for several cluster sizes: (a) $n=2$, (b) $n=3$, and (c) $n=4$. The symbols are experimental results [17]. 
thickness and for different clusters and initial energies.

Figure 10 depicts the stopping power ratio $R$ for $\mathrm{B}_{n}^{+}$, $n=23,6$, and 14 , as a function of the target thickness for different incident energies $\left(E_{0}=400,225,100\right.$, and $74 \mathrm{keV} /$ nucleon). The curves for $\mathrm{B}_{4}{ }^{+}$and $\mathrm{B}_{5}{ }^{+}$lie in between those for $\mathrm{B}_{3}{ }^{+}$and $\mathrm{B}_{6}{ }^{+}$and were not included in the figure for clarity. The first conclusion that comes out from this figure is that at large thicknesses the vicinage effects in the energy loss disappear. This is because Coulomb repulsion and nuclear scattering separate the fragments as the target thickness increases, decreasing the vicinage effect as was already shown in Figs. 6 and 7. Another observation is that the vicinage effects in the energy loss grow with the cluster size because each fragment feels the wake force of more particles as $n$ increases. Nevertheless this enhancement is not proportional to $n$ and the amount of $R$ gained by adding one more particle to the cluster tends to reduce as the cluster grows. For instance, the stopping power ratio $R$ is almost the same for $\mathrm{B}_{4}^{+}, \mathrm{B}_{5}^{+}$, and $\mathrm{B}_{6}^{+}$. This saturation in $R$ with $n$ is explained by the cancellation of the wake forces in large clusters. It is worth to notice that the decrease of $R$ at small thicknesses is due to the fact that, until charge equilibrium is reached, the amount of charge per atom is larger in $\mathrm{B}^{+}$than in $\mathrm{B}_{n}^{+}$, i.e. +1 versus $+1 / n$, respectively; and the stopping power increases with the projectile charge. The general conclusion that can be drawn from the analysis of Fig. 10 is that large clusters, high velocities, and thin foils are needed to measure large ratios (close to 2).

Our calculations are also compared with the available experimental results for $74 \mathrm{keV} /$ nucleon $\mathrm{B}_{2}{ }^{+}, \mathrm{B}_{3}{ }^{+}$, and $\mathrm{B}_{4}{ }^{+}$ molecular ions [17] in Fig. 11, showing a reasonable agreement considering the experimental error bars. There are also another experimental results concerning the energy loss of $\mathrm{B}_{3}$ neutral molecules in amorphous carbon foils [57], but they are not included in Fig. 11(b) because in this paper a differential stopping power was measured, which is different from the integral values that are usually measured [11-18] and we have calculated in this work.

\section{SUMMARY}

In this paper we have used a simulation code to analyze the interaction of swift $\mathrm{B}_{n}^{+}(n=2-6,14)$ clusters with thin amorphous carbon foils.
We have obtained that the fragments resulting from the cluster dissociation tend to separate each other inside the foil increasing the cluster size. But it is found that the size of the cluster in the direction of motion increases more than the corresponding size in the transversal direction, because the wake forces tend to align the fragments towards the direction of motion.

On the other hand, the widths of the angular and energy distributions are mainly governed by the Coulomb potential energy available when the cluster enters the foil, which is directly related to the cluster geometry and number of constituents. Therefore the width of the distributions grows with the number of components of the cluster. The wake forces have two clear effects: (i) they add an extra energy loss to the trailing fragments, lowering the mean value of the energy distributions, and (ii) they tend to align the trailing fragments into the direction of motion, producing a clearly visible peak at $0^{\circ}$ in the angular distributions. The elastic scattering, which is more important at lower energies, widens even more the angular distributions and washes out the alignment effect produced by the wake forces.

Finally we have observed that larger stopping power ratios $R$ are found for larger clusters with higher energies. The amount of $R$ that a cluster gains by increasing its number of constituents $n$ is not proportional to it. On the contrary there is a saturation in the increase of $R$ with $n$ due to the cancellation of wake forces in large clusters. The comparison with the experimental results [17] is rather good, with a reasonable agreement considering the experimental error bars.

\section{ACKNOWLEDGMENTS}

This work has been financially supported by the Spanish Ministerio de Educación y Ciencia (Contract Nos. BFM2003-04457-C02-01 and BFM2003-04457-C02-02). S.H.A. thanks the Fundación CajaMurcia for financial support and C.D.D. thanks the Spanish Ministerio de Educación y Ciencia for support under the Ramón y Cajal Program.
[1] R. Kelly and M. F. da Silva (eds.), Material Modification by High-Fluence Ion Beams, NATO ASI Series E: Applied Sciences Vol. 155 (Kluwer, Dordrecht, 1989).

[2] A. Gras-Martí, H. M. Urbassek, N. R. Arista, and F. Flores (eds.), Interaction of Charged Particles with Solids and Surfaces, NATO ASI Series B: Physics. Vol. 271 (Plenum, New York, 1991).

[3] M. A. Kumakhov and F. F. Komarov, Energy Loss and Ion Ranges in Solids (Gordon and Breach, New York, 1981).

[4] S. A. E. Johansson, J. L. Campbell, and K. G. Malmqvist, Particle Induced X-ray Emission Spectrometry PIXE (Wiley, New York, 1995).

[5] G. Kraft, Nucl. Instrum. Methods Phys. Res. A 454, 1 (2000).
[6] G. Kraft, Prog. Part. Nucl. Phys. 45, 5473 (2000).

[7] C. Deutsch and N. A. Tahir, Phys. Fluids B 4, 3735 (1992).

[8] E. Nardi, Z. Zinamon, and D. Ben-Hamu, Nuovo Cimento Soc. Ital. Fis., A 106A, 1839 (1993).

[9] G. Q. Wang, Y. N. Wang, and Z. L. Miškovic, Phys. Plasmas 12, 042702 (2005).

[10] N. R. Arista, Nucl. Instrum. Methods Phys. Res. B 164-165, 108 (2000).

[11] W. Brandt, A. Ratkowski, and R. H. Ritchie, Phys. Rev. Lett. 33, 1325 (1974).

[12] J. W. Tape, W. M. Gidson, J. Remillieux, R. Laubert, and H. E. Wegner, Nucl. Instrum. Methods 132, 75 (1976).

[13] R. Levi-Setti, K. Lam, and T. R. Fox, Nucl. Instrum. Methods 
Phys. Res. 194, 281 (1982).

[14] M. F. Steuer, D. S. Gemmell, E. P. Kanter, E. A. Johnson, and B. J. Zabransky, Nucl. Instrum. Methods Phys. Res. 194, 277 (1982).

[15] M. Farizon, N. V. de Castro Faria, B. Farizon-Mazuy, and M. J. Gaillard, Phys. Rev. A 45, 179 (1992).

[16] K. Baudin, A. Brunelle, M. Chabot, S. Della-Negra, J. Depauw, D. Gardès, P. Håkansson, Y. Le Beyec, A. Billebaud, M. Fallavier, J. Remillieux, J. C. Poizat, and J. P. Thomas, Nucl. Instrum. Methods Phys. Res. B 94, 341 (1994).

[17] K. Narumi, K. Nakajima, K. Kimura, M. Mannami, Y. Saitoh, S. Yamamoto, Y. Aoki, and H. Naramoto, Nucl. Instrum. Methods Phys. Res. B 135, 77 (1998).

[18] S. Yixiong, L. Xiting, J. Dongxing, and S. Dingyu, Nucl. Instrum. Methods Phys. Res. B 179, 480 (2001).

[19] A. Brunelle, S. Della-Negra, J. Depauw, D. Jacquet, Y. Le Beyec, and M. Pautrat, Phys. Rev. A 59, 4456 (1999).

[20] S. Heredia-Avalos, R. Garcia-Molina, and N. R. Arista, Europhys. Lett. 54, 729 (2001).

[21] R. Garcia-Molina, S. Heredia-Avalos, and I. Abril, J. Phys.: Condens. Matter 12, 5119 (2000).

[22] S. Heredia-Avalos, R. Garcia-Molina, and I. Abril, Nucl. Instrum. Methods Phys. Res. B 164-165, 296 (2000).

[23] A. Ricca and C. W. Bauschlicher, Jr., Chem. Phys. 208, 233 (1996).

[24] C. D. Denton, R. Garcia-Molina, I. Abril, and N. R. Arista, Nucl. Instrum. Methods Phys. Res. B 135, 50 (1998).

[25] R. Garcia-Molina, C. D. Denton, I. Abril, and N. R. Arista, Phys. Rev. A 62, 012901 (2000).

[26] R. Garcia-Molina, I. Abril, C. D. Denton, and S. HerediaAvalos, Nucl. Instrum. Methods Phys. Res. B 249, 6 (2006).

[27] M. P. Allen and D. J. Tildesley, Computer Simulation of Liquids (Oxford University Press, Oxford, 2002).

[28] D. Zajfman, G. Both, E. P. Kanter, and Z. Vager, Phys. Rev. A 41, 2482 (1990).

[29] P. L. Grande and G. Schiwietz, CasP version 3.1 (2004), code available at http://www.hmi.de/people/schiwietz/casp.html

[30] G. Schiwietz and P. L. Grande, Nucl. Instrum. Methods Phys. Res. B 175-177, 125 (2001).

[31] S. Heredia-Avalos and R. Garcia-Molina (unpublished).

[32] E. Bonderup, Penetration of Charged Particles through Matter: Lecture Notes (University of Aarhus, Aarhus, 1981).

[33] P. Sigmund, Particle Penetration and Radiation Effects: General Aspects and Stopping of Swift Point Charges, Springer Series in Solid-State Sciences Vol. 151 (Springer, Berlin, 2006).

[34] I. Abril, R. Garcia-Molina, C. D. Denton, F. J. Pérez-Pérez, and N. R. Arista, Phys. Rev. A 58, 357 (1998).

[35] S. Heredia-Avalos, J. C. Moreno-Marín, I. Abril, and R. Garcia-Molina, Nucl. Instrum. Methods Phys. Res. B 230, 118 (2005).

[36] S. Heredia-Avalos, R. Garcia-Molina, J. M. Fernández-Varea, and I. Abril, Phys. Rev. A 72, 052902 (2005).

[37] W. Brandt and M. Kitagawa, Phys. Rev. B 25, 5631 (1982).

[38] J. Cazaux and D. Gramari, J. Phys. (France) 38, L133 (1977).

[39] N. D. Mermin, Phys. Rev. B 1, 2362 (1970).

[40] U. Fano, Annu. Rev. Nucl. Sci. 13, 1 (1963).

[41] R. F. Egerton, Electron Energy-Loss Spectroscopy in the Electron Microscope (Plenum, New York, 1989).

[42] D. J. Planes, R. Garcia-Molina, I. Abril, and N. R. Arista, J. Electron Spectrosc. Relat. Phenom. 82, 23 (1996).

[43] E. Shiles, T. Sasaki, M. Inokuti, and D. Y. Smith, Phys. Rev. B 22, 1612 (1980).

[44] I. Abril, R. Garcia-Molina, N. R. Arista, and C. F. SanzNavarro, Nucl. Instrum. Methods Phys. Res. B 190, 89 (2002).

[45] J. C. Moreno-Marín, I. Abril, and R. Garcia-Molina, Nucl. Instrum. Methods Phys. Res. B 193, 30 (2002).

[46] ICRU Report 49, Stopping Powers and Ranges for Protons and Alpha Particles (International Commission on Radiation Units and Measurements, Bethesda, 1993).

[47] J. H. Ormrod and H. E. Duckworth, Can. J. Phys. 41, 1424 (1963).

[48] I. Hoffmann, E. Jäger, and U. Müller-Jahreis, Radiat. Eff. 31, 57 (1976).

[49] P. Mertens and Th. Krist, J. Appl. Phys. 53, 7343 (1982).

[50] Tao Zheng, Xiting Lu, Zonghuang Xia, and Dingyu Shen, Phys. Rev. B 57, 10213 (1998).

[51] C. Angulo, Th. Delbar, J.-S. Graulich, and P. Leleux, Nucl. Instrum. Methods Phys. Res. B 170, 21 (2000).

[52] Y. Zhang, Nucl. Instrum. Methods Phys. Res. B 196, 1 (2002); Y. Zhang, G. Possnert, and W. J. Weber, Appl. Phys. Lett. 80, 4662 (2002).

[53] W. Moller, G. Pospiech, and G. Schrieder, Nucl. Instrum. Methods 130, 265 (1975).

[54] J. F. Ziegler, J. P. Biersack, and U. Littmark, The Stopping and Ranges of Ions in Solids (Pergamon, New York, 1985), Vol. 1.

[55] R. Garcia-Molina, I. Abril, C. D. Denton, and N. R. Arista, Nucl. Instrum. Methods Phys. Res. B 164-165, 310 (2000).

[56] C. D. Denton, R. Garcia-Molina, I. Abril, and N. R. Arista, Nucl. Instrum. Methods Phys. Res. B 135, 55 (1998).

[57] D. Ben-Hamu, A. Baer, H. Feldman, J. Levin, O. Heber, Z. Amitay, Z. Vager, and D. Zajfman, Phys. Rev. A 56, 4786 (1997). 